\title{
Performance Efficiency of Quantization using HSV Colour Space and Vector Cosine Angle Distance in CBIR with Different Image Sizes
}

\author{
S.Niranjanan \\ Asst. Prof., Computer Science Department, \\ Mohamed Sathak College of Arts and Science, \\ Sholinganallur, Chennai-600 119
}

\begin{abstract}
Content Based Image Retrieval (CBIR) is an active research field in the past decades. Against the traditional system where the images are retrieved based on the key word search, CBIR systems retrieve the images based on the visual content. Even though some of the modern systems like relevance feedback system which improves the performance of CBIR systems exists, the importance of retrieving the images based on the low level features like Colour, Texture and Shape still determine the development of CBIR systems and cannot be undermined. Colour Histograms, Histogram Distance Measurements, Colour Spaces and Quantization play an important role in retrieving images based on similarities. In this paper, a novel method is presented for determining the efficiency of different quantization methods using HSV Colour space and measuring the Vector Cosine Angle distance of the images with different sizes of images like $256 \times 256$, $128 \times 128,64 \times 64,32$ X 32, 16 X 16 and 8 X 8 pixels for efficient image retrieval and comparing the time utilized for retrieval in each sizes and measuring the Overall efficiency.
\end{abstract}

\section{General Terms}

Content Based Image Retrieval (CBIR).

\section{Keywords}

Content Based Image Retrieval (CBIR), HSV Colour space, Vector Cosine Angle distance, quantization.

\section{INTRODUCTION}

Content Based Image Retrieval (CBIR) describes the content of the image using the visual features like Colour, Texture and Shape. It retrieves the images based on the visual contents rather than the traditional model of key word annotations of the contents. Even though it has many advantages, Italso has the major problem known as semantic Gap.In the semantic Gap, the description of the images using the low level features is unable to match the semantic intended by the user in his/her queries. Thus large number of false positive images are retrieved by the CBIR systems. The number of false positives are reduced when the spatial distribution of the visual features are integrated[1]. Even though some of the modern systems like relevance feedback system are introduced to improve the performance of CBIR exists, the importance of retrieving the images based on the low level features like Colour, Texture and Shape still determine the development of CBIR systems and cannot be undermined.In many cases they are the bottlenecks for development of CBIR techniques. A very basic issue in designing a CBIR system is to select the most effective image features for representing the image contents. Colour, Texture and Shape features are of the important and great majority primitive image descriptors in CBIR systems. Colour feature is one of the most reliable and easier visual

\author{
S.P.Raja Gopalan, PhD. \\ Prof., Dept. of Computer Science\& Engineering, \\ GKM College of Engineering \& Technology, \\ Chennai - 600063
}

features used in Image retrieval. It is robust to background complications and is independent of image size and orientation [18]. A lot of techniques available for retrieving images on the basis of Colour similarity from image database [3]. Colour Spaces, Colour Histograms, Histogram Distance Measurements, Size and Quantization play an important role in retrieving images based on similarities.

\section{COLOUR HISTOGRAM}

A colour histogram is a high dimensional feature vector, having greater than 100 dimensions. The comparison of histograms is computationally intensive. The colour histogram is widely used as an important colour feature indicating the contents of the images in content-based image retrieval (CBIR) systems. Especially, histogram-based algorithms are considered to be more effective for colour image indexing.

The global distribution of pixels of an image is described in Colour histogram, and it is insensitive to variations in scale and easy to calculate. The colour histogram for an image is constructed by counting the number of pixels of each colour. There are many difficulties with histogram based retrieval. The first of these is the high dimensionality of the colour histograms. Even with drastic quantization of the colour space, the image histogram feature spaces can occupy over 100 dimensions in real valued space. The large dimensionality also increases the complexity and computation of the distance function

An image histogram refers to the probability mass function of the image intensities. This is extended for Colour images to capture the joint probabilities of the intensities of the three Colour channels. More formally, the Colour histogram is defined by

$\mathrm{h}_{\mathrm{A}, \mathrm{B}, \mathrm{C}}(\mathrm{a}, \mathrm{b}, \mathrm{c})=\mathrm{N} \cdot \operatorname{Prob}(\mathrm{A}=\mathrm{a}, \mathrm{B}=\mathrm{b}, \mathrm{C}=\mathrm{c})$

where $A, B$ and $C$ represent the three Colour channels (R,G,B or $\mathrm{H}, \mathrm{S}, \mathrm{V})$ and $N$ is the number of pixels in the image. Computationally, the Colour histogram is formed by discretizing the Colours within an image and counting the number of pixels of each Colour.

Substantial quantization of the Colour space is needed, since the typical computer represents Colour images with up to 224 Colours. The main issues regarding the use of Colour histograms for indexing involve the choice of Colour space and correct quantization of the Colour space.When a perceptually uniform Colour space is chosen uniform quantization may be appropriate. If a non-uniform Colour space is chosen, then non-uniform quantization may be needed.

The HSV Colour space offers improved perceptual uniformity. It represents with equal emphasis the three Colour 
variants that characterize Colour: Hue, Saturation and Value (Intensity). This separation is attractive because Colour image processing performed independently on the Colour channels does not introduce false Colours.

There are several distance formulas for measuring the similarity of Colour histograms. The Colour distance formulas arrive at a measure of similarity between images based on the perception of Colour content. Three distance formulas that are used normally for image retrieval are histogram Euclidean Distance, histogram IntersectionDistance and histogram Quadratic (cross) Distance [13]. There are many other histogram distance formulas like Vector Cosine Angle Distance, Correlation Distance and Spearman Distance etc.,.

\section{HISTOGRAM VECTOR COSINE ANGLEDISTANCE}

If two vectors $X$ and $Y$ are considered, where $X \equiv\left(x_{1}, x_{2}\right.$, $\left.\ldots \ldots x_{n}\right)$ and $Y \equiv\left(y_{1}, y_{2}, \ldots . y_{n}\right)$, then $\cos \Theta$ may be considered as the Cosine of the vector angle between $\mathrm{X}$ and $\mathrm{Y}$ in $\mathrm{n}$ dimension. Formally, Vector Cosine Angle Distance is defined as follows.

$\mathrm{VCAD}=\frac{\sum_{i} x_{i} y_{i}}{\sqrt{\sum_{i} x_{i}^{2}} \sqrt{\sum_{i} y_{i}^{2}}}=\frac{X, Y}{\|X\|\|Y\|}$

The Vector Cosine Angle Distance between two 2dimensional vectors $\left(\mathrm{X}_{1}, \mathrm{X}_{2}\right)$ and $\left(\mathrm{Y}_{1}, \mathrm{Y}_{2}\right)$ is as shown below

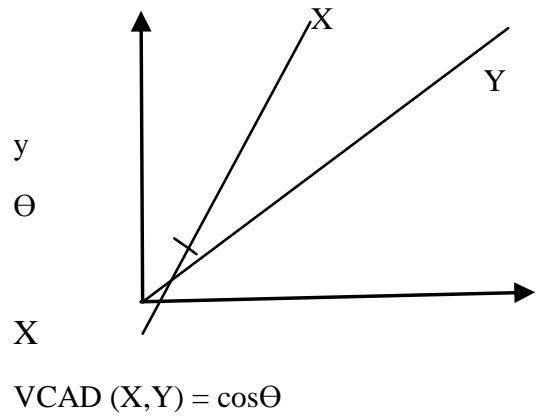

One important property of Vector Cosine Angle is that it gives a metric of similarity between two vectors. This makes it easy to combine distance between two images using multiple features.

Count the number of pixels that correspond to a specific Colour in quantized Colour space for computation of histogram. In order to compare histograms of two images, first specific codes for all histogram bins are to be generated.

\section{COLOURSPACE QUANTIZATION}

A Color quantization is a process that reduces the number of distinct colors used in an image.The intention of Colour quantization is that the new image should be as visually similar as possible to the original image. For a true color image, the number of the kind of colors are up to $2^{24}=$ 16777216, so the direct extraction of color feature from true color will lead to a large computation. In order to reduce the computation, without a significant reduction in image quality, some representative color is extracted, to represent the image, thereby reducing the storage space and enhancing the process speed [5]. The effect of color quantization on the performance of image retrieval has been reported by many authors in [14] [16] [17] and [20], with different quantization schemes, like RGB (8X8X8), Lab (4X8X8), HSV (16X4X4), Lu*v* (4X8X8).

ManimalaSinglia [8] concluded that, the HSV quantization $(16,4,4)$ with Histogram Intersection Distance gives best performance then the other colour spaces and the averageretrieval time of HSV Colour space gives the optimum value, considering the precision and recall values. Bo Di [4] quantized HSV Colour space, 19 hues step $20(0,20,40, \ldots$, $360), 4$ saturations, step $0.25(0.173,0.423,0.673,0.923)$ and 5 values, step $0.25(0,0.25,0.5,0.75,1.00)$. Muhammad Riaz et al [9] defined: 18 hues, step $20(350,10,30,50 \ldots 350), 5$ saturations, step $0.2(0,0.2,0.4,0.6,0.8,1.0)$ and 3 values, steps $0.25(0,0.5,0.75,1.0)$.Ch. Kavitha et al [6],[7] defined 8 hues, step (316-20, 21-40, 41-75, 76-155, 156-190, 191-270, 271-295 and 296-315), 3 saturations, step (0-0.2, 0.2-0.7 and $0.7-1.0)$ and 3 values, step (0-0.2, 0.2-0.7 and 0.7-1.0) and concluded that colour and texture features of image sub blocks combined with normalized Euclidean distance yields better performance results.M.BabuRao et al [2] defined 8 hues, 3 saturations and 3 values.Wasim Khan et al [19] defined 18 hues, 3 saturations and 3 values.SangohJeong [13] used 18 hues, 3 saturations, 3 values and concluded that the Histogram Intersection-based image retrieval in HSV Colour space is most desirable among six retrieval methods including histogram euclidean distance in RGB Colour space, histogram intersection in RGB Colour Space, histogram quadratic (cross) distance in RGB Colour Space, histogram euclidean distance in HSV Colour space, histogram intersection in HSV Colour Space, histogram quadratic (cross) distance in HSV Colour Space mentioned in considering both computation time and retrieval effectiveness. RajshreeDubey et al [12] have found that the histogram Euclidean distance and histogram intersection distance in HSV Colour space are most useful among histogram distance measures in the average sense. Vishal Chitkara [15] found that Variable-Bin Allocation (VBA) performs better than Constant-Bin Allocation (CBA) and the VBA approach not only performs better, but also consumes less resources, which is an important feature.Niranjanan et al [10], [11] concluded that the HSV Colour Space Quantization ( 2, 2, 2 ) with Histogram Euclidean Distance and ( 2, 2, 10 ) with Histogram Intersection Distance under a uniform size of 256 X 256 pixels gives better performance.

\section{PROPOSED MODEL}

\subsection{Methodology}

In this paper the issue of image database retrieval based on Colour using HSV colour space is addresed. Histogram search characterizes an image by its Colour distribution. Before extracting the maximum Colour from each segment the input image is converted to HSV and adaptive segmentation is applied on the HSV Colour space. This will compute the feature vector. Different quantization of hue, saturation and value are used. In this experiment, codes were generated for 10 different hue steps, 3 different saturations steps and 3 
different value steps (10 H x $3 \mathrm{~S}$ x $3 \mathrm{~V}$ ) $90 \mathrm{HSV}$ histogram models. Accordingly codes were generated for more Histogram bins and then, histogram Vector Cosine Angle distances were computed in each quantized Colour space. Minkowski - Form Distance Matrics is used to calculate the distance.

The corresponding image is first resized todifferent sizes of images like 256 X 256, 128 X 128, 64 X 64, 32 X 32, 16 X 16 and $8 \times 8$ pixels, then converted from RGB to HSV Colour space using known equations, and then it is partitioned into $\mathrm{m}$ number of areas based on different ranges of hue, saturation and value. In the next step each area is partitioned into $n$ number of segments based on the number of pixels it contains. After getting those segments the maximum Colour occurrence in each segment is calculated using the hue histogram information and this is used as a feature vector. Finally, the feature vector for query image is compared with the feature vector of the database images. The proposed system has been implemented and verified

\subsection{Maximum Colour Selection}

The images are converted from RGB to HSV using the conversion formula as follows

$H=\cos ^{-1}\left\{\frac{1 / 2[(R-G)+(R-B)]}{\sqrt{(R-G)^{2}+(R-B)(G-B)}}\right\}$

$S=1-\frac{3}{R+G+B}[\min (R, G, B)]$

$V=\frac{1}{3}(R+G+B)$

After the execution of these equations $\mathrm{H}, \mathrm{S}$ and $\mathrm{V}$ will have values $0-255$. To convert hue into angle that ranges from $0^{\circ}$ to $360^{\circ}$, value and saturation from 0 to 1 following equations are used

$\mathrm{H}=((\mathrm{H} / 255) * 360) \bmod 360$

$\mathrm{V}=\mathrm{V} / 255$

$\mathrm{S}=\mathrm{S} / 255$

After conversion from RGB to HSV the next design parameter is the quantization of the Colour space. The HSV Colour space is cylindrical. The long axis represents value: blackness to whiteness. Distance from the axis represents saturation: amount of Colour present. The angle around the axis is the hue: tint or tone. Since hue represents the most significant characteristic of the Colour, it requires the finest quantization. In the hue circle the primaries red, green and blue are separated by 120 degrees.

A large number of images have been tested for deciding which step could yield best results and made different steps in Hue, Saturation and Value. In this experiment, 10 Hue models with various steps were designed which varies from 5to 180 hues, Step 5 ( $355,360,5,10,15, \ldots 355)$, Step 10 ( 350, $360,10,20, \ldots 350)$, Step $15(345,360,15,30, .345)$, Step $20(340,360,20,40, \ldots 340)$, Step $24(336,360,24,48, \ldots$ $336)$, Step $30(330,360,30,60, \ldots 330)$, Step 60 ( 300, 360, $60, \ldots 360)$, Step $90(270,360,90, \ldots 270)$, Step 120 ( 240, $360,120,240)$ and Step $180(180,360,180)$ ).Also 3 Saturation models with various steps were defined which varies from 2 to 10 Saturations, Step 0.5 ( 0, 0.5, 1.0$)$, Step $0.2(0,0.2,0.4,0.6,0.8,1.0)$ and Step $0.1(0,0.1,0.2, \ldots$ 1.0).In the same way 3 Values models with various steps were defined which varies from 2 to 10 Values, Step 0.5 ( $0,0.5$, $1.0)$, Step $0.2(0,0.2,0.4,0.6,0.8,1.0)$ and Step $0.1(0,0.1$, $0.2, \ldots 1.0$.

As a result, a large array of $2 * 2 * 2=8$ to $72 * 10 * 10=7,200$ number of different areaas are available, each representing different Colour distribution of the image.After partitioning into $m$ number of areas, HSV information spaced image is further divided into $n$ number of segments depending upon the number of pixels in each area. The areas which have more pixels are partitioned into more number of segments and the areas which have fewer pixels are divided into less number of segments. Through this procedure the distribution characteristics for the Colour tone distributed in the corresponding image is got. After extracting this Colour distribution information from the image, the maximum Colour occurrence in each segment is calculated by using hue histogram of the image and HSV value of that pixel which is used to make a feature vector. Such operations are carried out for the entire image and then feature vector is computed.

Following equation is used to partition each area in n number of segments; $\mathrm{n}$ can have different values for different areas.

$n_{i=\left(\left(X_{i} / T\right)\right) * T S} \quad 0<i<=m$

Wheren ${ }_{i}$ represents the number of segments in area $i$, ${ }_{X i}$ represents the number of pixels in area $\mathrm{i}$ (where iranges from 1 to $m$ ), $T$ represents total number of pixels of the image and $T S$ represents total number of required segments of the entire HSV image. After executing the equation huehistogram is computed for each segment. From that histogram, the each Colour occurrence in that segmentcanbe easily detected. The maximum Colour occurrence from each segment is selected and used its HSV value to compute the feature vector. This feature vector is used for comparison with the images in the database and matching for each image is calculated using Minkowski Metric (LM norm) equation. Segments in each area of the query image are compared with the corresponding area of the database image and for this comparison Minkowski Metric is used as shown below:

$d_{M(i, j)=\left(\sum_{k=1}^{P}\left|\left(x_{i}^{k}-x_{j}^{k}\right)\right|\right)^{1 / M}}$

Where, $\mathrm{p}$ is the dimension of the vector $x_{i}^{k}$ is the $k^{\text {th }}$ element of $x_{i}$. Three special cases of the LM metric are of particular interest, namely, $\mathrm{L}=1,2, \infty$.

A Matlab program using these different quantization schemes is programmed to retrieve images from a fixed size data base using a query image and converting the images to the size equivalent to the Query Image and comparing them. The time consumed for displaying the resultant images is stored in a database along with the quantization variants data. The screen shots of retrieved images are also stored with appropriate names.

\subsection{Algorithm For Proposed Scheme}

Step 1: Load database in the Mat lab workspace.

Step 2: Resize the image for appropriate size like [256,256], [128,128], [64,64], [32,32], [16,16] and [8,8].

Step 3: Convert image from RGB to HSV.

Step 4: Generate the histogram of hue, saturation and value

Step 5: Generate no. of signatures $n$ for hue, $m$ for saturation and $x$ for value. 
Step6: Store the signature of database images into the mat file

Step 7: Load the Query image.

Step 8: Apply the procedure 2-7 to find signature of Query image.

Step 9: Determine the normalized Vector Cosine Angle distance of signature of Query image with stored signature of database.

Step 10: Sort the normalized Vector Cosine Angle distance values to perform indexing.

Step 11: Display the result on GUI.

Step 12: Create a folder in the name of corresponding variants

Step 13: Create a file in the name of corresponding variants \& save

Step 14: Apply the procedure 2-13 for various Hue, Saturation and Value steps

\subsection{Performance Evaluation}

The performance of retrieval system can be measured in terms of its recall and precision. Recall measure the ability of the system to retrieve all the models that are relevant, while precision measures the ability of the system to retrieve only models that are relevant. This histogram has a high recall and precision of retrieval, and is effectively used in content-based image retrieval systems. They are defined as:

Precision $=\frac{\text { Number of relevant images retrieved }}{\text { Total number of images retrieved }}=\frac{A}{A+B}$

Recall $=\frac{\text { Number of relevant images retrieved }}{\text { Total number of relevant images }}=\frac{A}{A+C}$

Where A represent the number of relevant images that are retrieved, $\mathrm{B}$, the number of irrelevant items and the $\mathrm{C}$, number of relevant items those were not retrieved. The number of relevant items retrieved is the number of the returned images that are similar to the query image in this case. The number of relevant items in collection is the number of images that are in the same particular category with the query image. The total number of items retrieved is the number of images that are returned by the search engine.

\section{EXPERIMENTAL RESULTS}

Only 7 relevant images of the particular query image typehave been purposefully kept in the database folder along with other irrelevant images. A $3 \times 3$ matrixis designed for showing the resultant retrieved images in the result window. Out of the total 9 images in the resultant matrix only 7 relevant images can be retrieved by the program and the other two images are selected based on the nearest Vector Cosine Angle distance.
Hence the combination of different Hue, Saturation and Value steps which achieved retrieving all the 7 relevant images from the database folder in the least time is the best possible combination for image retrieval in HSV colour space. In the present Vector Cosine Angle Distance, none of the Quantization variants retrieved all the 7 relevant images. Hence, the next better possibility of retrieving 6 relevant images out of 7 relevant images was considered.

Table 1 contains all the combinations of Hue, Saturation and Value steps for all different sizes of images which resulted in retrieving 6 out of all the 7 possible relevant images from the database folder and whose Recall value is equal to 0.857143 .

The present work with the result of HSV Colour Space Quantization with Vector Cosine Angle Distancewith least time consumption in different sizes as listed below gives a better alternative than other Quantization schemes.

In 256 X 256 pixel images, the HSV slice $(2,10,2)$ recovered the Images in 35.6142Secs.

In $128 \mathrm{X} 128$ pixel images, the HSV slice $(2,10,2)$ recovered the Images in 10.2485 Secs.

In $64 \mathrm{X} 64$ pixel images, the HSV slice $(2,5,2)$ recovered the Images in 2.9226 Secs.

In $32 \times 32$ and $16 \times 16$ pixel images, no HSV slice had recovered 6 relevant Images out of 7 . Only 5 relevant images out of 7 are recovered and hence not considered.

In $8 \times 8$ pixel images, the HSV slice $(2,5,10)$ recovered the Images in 1.7708 Secs.

\section{CONCLUSION}

The above results show that it can be possible to retrieve 6 relevant images out of 7 of uniform size $8 \times 8$ pixels from the databases by adopting only 2 slices of Hue (i.e. $0-180$ and $180-360$ ), 5 slices of Saturation (i.e. 0 - 0.2, $0.2-0.4,0.4-$ $0.6,0.6-0.8$ and $0.8-1.0$ ) and 10 slices of Value (i.e. $0-0.1$, $0.1-0.2,0.2-0.3,0.3-0.4,0.4-0.5,0.5-0.6,0.6-0.7,0.7$ $-0.8,0.8-0.9$ and $0.9-1.0$ ) in 1.7708Secs than the other sizes like 16 X 16, 32 X 32, 64 X 64, 128 X 128 and 256 X 256.

Thus 2 X 5 X $10=100$ slices split in $8 \times 8$ pixel size is more than sufficient to retrieve 6 relevant imagesout of 7 in much lesser Time over other sizes. Thus it is concluded that the HSV colour space Quantization $(2,5,10)$ with Histogram Vector Cosine Angle distance under a uniform size of 8 X 8 pixels gives better performance than other variations of Hue, Saturation and Value quantization models in image retrieval. 
HSV Experimental Image Results of Least Time Consuming Quantization Variants

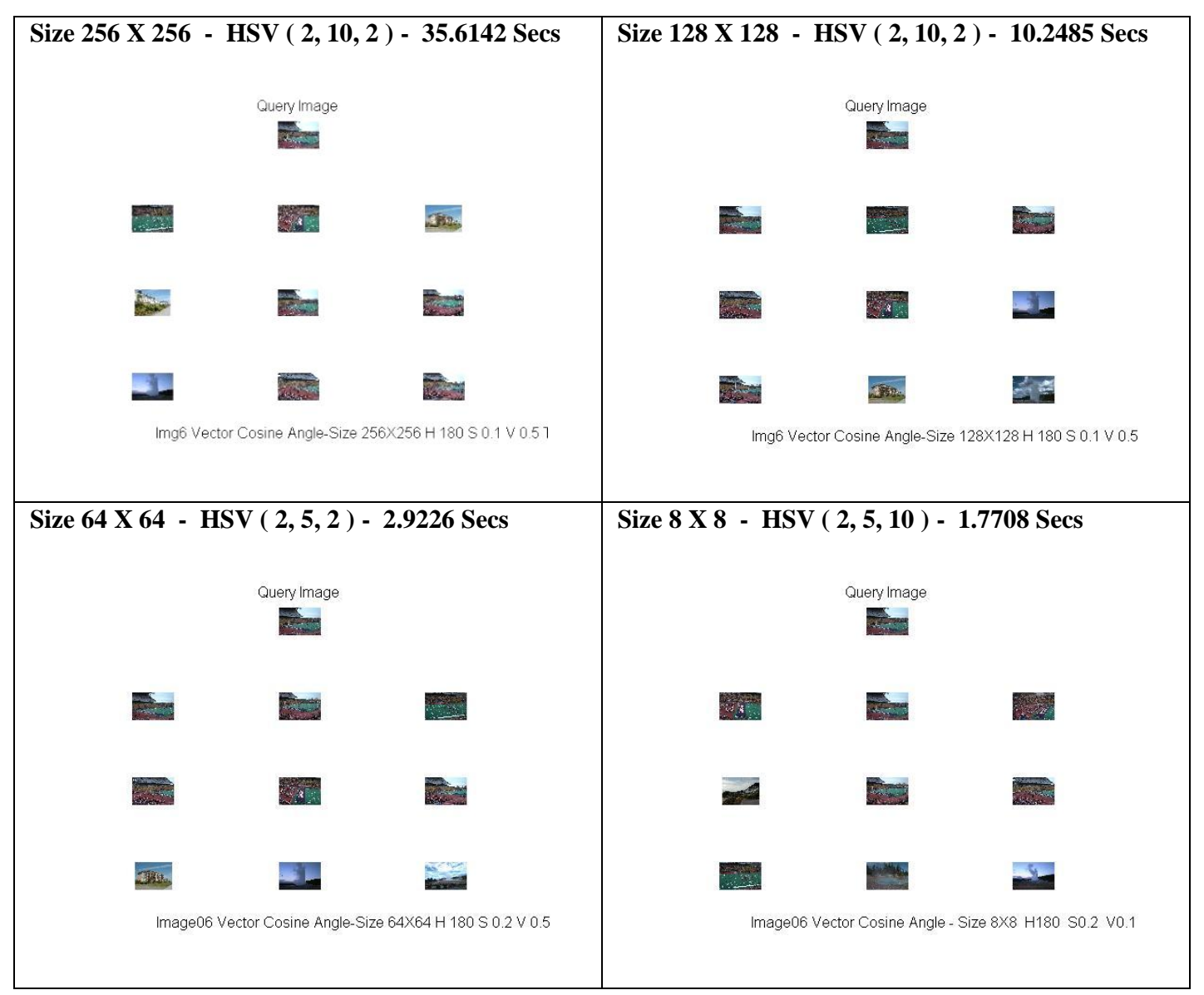




\section{HSV Experimental Results of Most Desirable Quantization Variants}

Full Performance in Intel i7 with 16 GB RAM

Table 1 Results where Recall $=\mathbf{0 . 8 5 7 1 4 3}$, Precision $=\mathbf{0 . 6 6 6 6 6 7}$ and retrieved 6 relevant images out of 7 .

\begin{tabular}{|c|c|c|c|c|c|c|c|c|c|c|}
\hline \multicolumn{4}{|c|}{ Hue, Saturation and Value steps } & \multirow[t]{2}{*}{$\begin{array}{l}\text { Hue, } \\
\text { Saturation } \\
\text { and Value } \\
\text { Slices }\end{array}$} & \multicolumn{6}{|c|}{$\begin{array}{l}\text { Vector Cosine Angle Distance Comparison ( Time in Secs ) with } \\
\text { Different Sizes Where Recall }=0.857143 \text { (Only } 6 \text { out of } 7 \\
\text { Available Relevant Images Recovered out of Total of } 9 \text { Images } \\
\text { Including } 2 \text { Irrelevant Images) }\end{array}$} \\
\hline $\begin{array}{l}\text { SL. } \\
\text { NO. }\end{array}$ & $\begin{array}{l}\text { HUE } \\
\text { Step }\end{array}$ & $\begin{array}{l}\text { SATURATION } \\
\text { Step }\end{array}$ & $\begin{array}{l}\text { VALUE } \\
\text { Step }\end{array}$ & & $\begin{array}{l}256 X \\
256\end{array}$ & $\begin{array}{l}128 X \\
128\end{array}$ & $64 \times 64$ & $32 \times 32$ & $16 \times 16$ & $8 \times 8$ \\
\hline 1 & 5 & 0.1 & 0.1 & $72,10,10$ & NA & NA & NA & NA & NA & NA \\
\hline 2 & 5 & 0.1 & 0.2 & $72,10,5$ & NA & NA & 33.064 & NA & NA & NA \\
\hline 3 & 5 & 0.1 & 0.5 & $72,10,2$ & 877.1498 & 220.0204 & 54.0178 & NA & NA & NA \\
\hline 4 & 5 & 0.2 & 0.1 & $72,5,10$ & NA & NA & NA & NA & NA & 1.9808 \\
\hline 5 & 5 & 0.2 & 0.2 & $72,5,5$ & NA & NA & NA & NA & NA & NA \\
\hline 6 & 5 & 0.2 & 0.5 & $72,5,2$ & NA & NA & 29.1827 & NA & NA & NA \\
\hline 7 & 5 & 0.5 & 0.1 & $72,2,10$ & NA & NA & NA & NA & NA & NA \\
\hline 8 & 5 & 0.5 & 0.2 & $72,2,5$ & NA & NA & $\mathrm{NA}$ & NA & NA & $\mathrm{NA}$ \\
\hline 9 & 5 & 0.5 & 0.5 & $72,2,2$ & NA & NA & NA & $\mathrm{NA}$ & NA & NA \\
\hline 10 & 10 & 0.1 & 0.1 & $36,10,10$ & NA & NA & NA & NA & NA & NA \\
\hline 11 & 10 & 0.1 & 0.2 & $36,10,5$ & NA & NA & 18.1171 & NA & NA & NA \\
\hline 12 & 10 & 0.1 & 0.5 & $36,10,2$ & 444.5443 & 111.963 & 28.1321 & NA & NA & NA \\
\hline 13 & 10 & 0.2 & 0.1 & $36,5,10$ & NA & NA & NA & NA & NA & 1.8662 \\
\hline 14 & 10 & 0.2 & 0.2 & $36,5,5$ & NA & NA & $\mathrm{NA}$ & NA & NA & NA \\
\hline 15 & 10 & 0.2 & 0.5 & $36,5,2$ & $\mathrm{NA}$ & NA & 15.7158 & NA & NA & NA \\
\hline 16 & 10 & 0.5 & 0.1 & $36,2,10$ & NA & NA & NA & NA & NA & NA \\
\hline 17 & 10 & 0.5 & 0.2 & $36,2,5$ & NA & NA & NA & NA & NA & $\mathrm{NA}$ \\
\hline 18 & 10 & 0.5 & 0.5 & $36,2,2$ & NA & NA & NA & NA & NA & NA \\
\hline 19 & 15 & 0.1 & 0.1 & $24,10,10$ & NA & NA & NA & NA & NA & NA \\
\hline 20 & 15 & 0.1 & 0.2 & $24,10,5$ & NA & NA & 13.2388 & NA & NA & NA \\
\hline 21 & 15 & 0.1 & 0.5 & $24,10,2$ & 299.9656 & 76.3728 & 19.5651 & NA & NA & $\mathrm{NA}$ \\
\hline 22 & 15 & 0.2 & 0.1 & $24,5,10$ & NA & NA & NA & NA & NA & 1.8198 \\
\hline 23 & 15 & 0.2 & 0.2 & $24,5,5$ & NA & NA & $\mathrm{NA}$ & NA & NA & NA \\
\hline 24 & 15 & 0.2 & 0.5 & $24,5,2$ & NA & NA & 11.1691 & NA & NA & NA \\
\hline 25 & 15 & 0.5 & 0.1 & $24,2,10$ & NA & NA & NA & NA & NA & NA \\
\hline 26 & 15 & 0.5 & 0.2 & $24,2,5$ & NA & NA & $\mathrm{NA}$ & NA & NA & $\mathrm{NA}$ \\
\hline 27 & 15 & 0.5 & 0.5 & $24,2,2$ & NA & NA & NA & NA & NA & NA \\
\hline 28 & 20 & 0.1 & 0.1 & $18,10,10$ & NA & NA & NA & NA & NA & NA \\
\hline 29 & 20 & 0.1 & 0.2 & $18,10,5$ & NA & NA & 10.6704 & NA & NA & NA \\
\hline 30 & 20 & 0.1 & 0.5 & $18,10,2$ & 228.142 & 58.2995 & 15.3107 & NA & NA & NA \\
\hline 31 & 20 & 0.2 & 0.1 & $18,5,10$ & NA & NA & NA & NA & NA & 1.804 \\
\hline 32 & 20 & 0.2 & 0.2 & $18,5,5$ & NA & NA & NA & NA & NA & NA \\
\hline \multicolumn{4}{|c|}{ Hue, Saturation and Value steps } & Hue, & \multicolumn{6}{|c|}{ Vector Cosine Angle Distance Comparison ( Time in Secs ) with } \\
\hline
\end{tabular}




\begin{tabular}{|c|c|c|c|c|c|c|c|c|c|c|}
\hline \multirow[b]{2}{*}{$\begin{array}{l}\text { SL. } \\
\text { NO. }\end{array}$} & \multirow[b]{2}{*}{$\begin{array}{l}\text { HUE } \\
\text { Step }\end{array}$} & \multirow[b]{2}{*}{$\begin{array}{l}\text { SATURATION } \\
\text { Step }\end{array}$} & \multirow[b]{2}{*}{$\begin{array}{l}\text { VALUE } \\
\text { Step }\end{array}$} & \multirow[t]{2}{*}{$\begin{array}{l}\text { Saturation } \\
\text { and Value } \\
\text { Slices }\end{array}$} & \multicolumn{6}{|c|}{$\begin{array}{c}\text { Different Sizes Where Recall }=0.857143 \text { (Only } 6 \text { out of } 7 \\
\text { Available Relevant Images Recovered out of Total of } 9 \text { Images } \\
\text { Including } 2 \text { Irrelevant Images ) }\end{array}$} \\
\hline & & & & & $\begin{array}{l}256 X \\
256\end{array}$ & $\begin{array}{l}128 X \\
128\end{array}$ & $64 \times 64$ & $32 \times 32$ & $16 \times 16$ & $8 \times 8$ \\
\hline 33 & 20 & 0.2 & 0.5 & $18,5,2$ & NA & NA & 8.935 & NA & NA & NA \\
\hline 34 & 20 & 0.5 & 0.1 & $18,2,10$ & NA & NA & NA & $\mathrm{NA}$ & $\mathrm{NA}$ & NA \\
\hline 35 & 20 & 0.5 & 0.2 & $18,2,5$ & $\mathrm{NA}$ & $\mathrm{NA}$ & $\mathrm{NA}$ & $\mathrm{NA}$ & $\mathrm{NA}$ & $\mathrm{NA}$ \\
\hline 36 & 20 & 0.5 & 0.5 & $18,2,2$ & NA & NA & NA & NA & NA & NA \\
\hline 37 & 24 & 0.1 & 0.1 & $15,10,10$ & $\mathrm{NA}$ & NA & NA & NA & NA & NA \\
\hline 38 & 24 & 0.1 & 0.2 & $15,10,5$ & $\mathrm{NA}$ & NA & 9.4747 & $\mathrm{NA}$ & $\mathrm{NA}$ & NA \\
\hline 39 & 24 & 0.1 & 0.5 & $15,10,2$ & 192.3997 & 49.2683 & 13.1293 & NA & NA & NA \\
\hline 40 & 24 & 0.2 & 0.1 & $15,5,10$ & NA & NA & NA & NA & NA & 1.8146 \\
\hline 41 & 24 & 0.2 & 0.2 & $15,5,5$ & $\mathrm{NA}$ & $\mathrm{NA}$ & $\mathrm{NA}$ & NA & $\mathrm{NA}$ & NA \\
\hline 42 & 24 & 0.2 & 0.5 & $15,5,2$ & NA & NA & 7.8149 & NA & NA & NA \\
\hline 43 & 24 & 0.5 & 0.1 & $15,2,10$ & NA & NA & NA & NA & NA & NA \\
\hline 44 & 24 & 0.5 & 0.2 & $15,2,5$ & NA & NA & NA & NA & NA & $\mathrm{NA}$ \\
\hline 45 & 24 & 0.5 & 0.5 & $15,2,2$ & NA & NA & NA & NA & NA & NA \\
\hline 46 & 30 & 0.1 & 0.1 & $12,10,10$ & NA & NA & NA & NA & NA & NA \\
\hline 47 & 30 & 0.1 & 0.2 & $12,10,5$ & $\mathrm{NA}$ & NA & 8.2849 & $\mathrm{NA}$ & NA & NA \\
\hline 48 & 30 & 0.1 & 0.5 & $12,10,2$ & 155.868 & 40.3478 & 11.0477 & NA & NA & NA \\
\hline 49 & 30 & 0.2 & 0.1 & $12,5,10$ & NA & NA & NA & NA & NA & 1.7984 \\
\hline 50 & 30 & 0.2 & 0.2 & $12,5,5$ & $\mathrm{NA}$ & $\mathrm{NA}$ & $\mathrm{NA}$ & NA & NA & NA \\
\hline 51 & 30 & 0.2 & 0.5 & $12,5,2$ & NA & NA & 6.6919 & NA & NA & NA \\
\hline 52 & 30 & 0.5 & 0.1 & $12,2,10$ & NA & NA & NA & NA & NA & NA \\
\hline 53 & 30 & 0.5 & 0.2 & $12,2,5$ & NA & NA & NA & NA & NA & NA \\
\hline 54 & 30 & 0.5 & 0.5 & $12,2,2$ & $\mathrm{NA}$ & $\mathrm{NA}$ & $\mathrm{NA}$ & $\mathrm{NA}$ & $\mathrm{NA}$ & $\mathrm{NA}$ \\
\hline 55 & 60 & 0.1 & 0.1 & $6,10,10$ & NA & $\mathrm{NA}$ & $\mathrm{NA}$ & NA & NA & $\mathrm{NA}$ \\
\hline 56 & 60 & 0.1 & 0.2 & $6,10,5$ & $\mathrm{NA}$ & NA & 6.1834 & NA & NA & NA \\
\hline 57 & 60 & 0.1 & 0.5 & $6,10,2$ & 83.7707 & 22.1966 & 6.7446 & $\mathrm{NA}$ & $\mathrm{NA}$ & NA \\
\hline 58 & 60 & 0.2 & 0.1 & $6,5,10$ & NA & NA & NA & $\mathrm{NA}$ & NA & 1.7809 \\
\hline 59 & 60 & 0.2 & 0.2 & $6,5,5$ & NA & NA & NA & NA & NA & NA \\
\hline 60 & 60 & 0.2 & 0.5 & $6,5,2$ & $\mathrm{NA}$ & $\mathrm{NA}$ & 4.401 & $\mathrm{NA}$ & $\mathrm{NA}$ & $\mathrm{NA}$ \\
\hline 61 & 60 & 0.5 & 0.1 & $6,2,10$ & NA & NA & $\mathrm{NA}$ & NA & NA & $\mathrm{NA}$ \\
\hline 62 & 60 & 0.5 & 0.2 & $6,2,5$ & $\mathrm{NA}$ & NA & $\mathrm{NA}$ & NA & NA & $\mathrm{NA}$ \\
\hline 63 & 60 & 0.5 & 0.5 & $6,2,2$ & $\mathrm{NA}$ & $\mathrm{NA}$ & $\mathrm{NA}$ & NA & NA & $\mathrm{NA}$ \\
\hline 64 & 90 & 0.1 & 0.1 & $4,10,10$ & $\mathrm{NA}$ & $\mathrm{NA}$ & $\mathrm{NA}$ & $\mathrm{NA}$ & $\mathrm{NA}$ & $\mathrm{NA}$ \\
\hline 65 & 90 & 0.1 & 0.2 & $4,10,5$ & $\mathrm{NA}$ & NA & 4.9202 & NA & NA & NA \\
\hline 66 & 90 & 0.1 & 0.5 & $4,10,2$ & 59.6017 & 16.3202 & 5.196 & $\mathrm{NA}$ & $\mathrm{NA}$ & NA \\
\hline 67 & 90 & 0.2 & 0.1 & $4,5,10$ & NA & NA & NA & NA & NA & 1.774 \\
\hline 68 & 90 & 0.2 & 0.2 & $4,5,5$ & NA & NA & NA & NA & NA & NA \\
\hline 69 & 90 & 0.2 & 0.5 & $4,5,2$ & $\mathrm{NA}$ & $\mathrm{NA}$ & 3.6796 & $\mathrm{NA}$ & $\mathrm{NA}$ & $\mathrm{NA}$ \\
\hline 70 & 90 & 0.5 & 0.1 & $4,2,10$ & NA & NA & NA & NA & NA & NA \\
\hline \multicolumn{4}{|c|}{ Hue, Saturation and Value steps } & Hue, & \multicolumn{6}{|c|}{ Vector Cosine Angle Distance Comparison ( Time in Secs ) with } \\
\hline
\end{tabular}




\begin{tabular}{|c|c|c|c|c|c|c|c|c|c|c|}
\hline \multirow[b]{2}{*}{$\begin{array}{l}\text { SL. } \\
\text { NO. }\end{array}$} & \multirow[b]{2}{*}{$\begin{array}{l}\text { HUE } \\
\text { Step }\end{array}$} & \multirow[b]{2}{*}{$\begin{array}{l}\text { SATURATION } \\
\text { Step }\end{array}$} & \multirow[b]{2}{*}{$\begin{array}{l}\text { VALUE } \\
\text { Step }\end{array}$} & \multirow[t]{2}{*}{$\begin{array}{l}\text { Saturation } \\
\text { and Value } \\
\text { Slices }\end{array}$} & \multicolumn{6}{|c|}{$\begin{array}{c}\text { Different Sizes Where Recall }=0.857143 \text { (Only } 6 \text { out of } 7 \\
\text { Available Relevant Images Recovered out of Total of } 9 \text { Images } \\
\text { Including } 2 \text { Irrelevant Images) }\end{array}$} \\
\hline & & & & & $\begin{array}{l}256 X \\
256\end{array}$ & $\begin{array}{l}128 X \\
128\end{array}$ & $64 \times 64$ & $32 \times 32$ & $16 \times 16$ & $8 \times 8$ \\
\hline 71 & 90 & 0.5 & 0.2 & $4,2,5$ & NA & NA & NA & NA & NA & NA \\
\hline 72 & 90 & 0.5 & 0.5 & $4,2,2$ & NA & NA & NA & NA & NA & NA \\
\hline 73 & 120 & 0.1 & 0.1 & $3,10,10$ & NA & NA & NA & $\mathrm{NA}$ & NA & NA \\
\hline 74 & 120 & 0.1 & 0.2 & $3,10,5$ & NA & NA & 4.4624 & NA & NA & NA \\
\hline 75 & 120 & 0.1 & 0.5 & $3,10,2$ & 47.6637 & 13.2069 & 4.4755 & NA & NA & NA \\
\hline 76 & 120 & 0.2 & 0.1 & $3,5,10$ & $\mathrm{NA}$ & NA & NA & NA & NA & 1.7714 \\
\hline 77 & 120 & 0.2 & 0.2 & $3,5,5$ & NA & NA & NA & $\mathrm{NA}$ & NA & NA \\
\hline 78 & 120 & 0.2 & 0.5 & $3,5,2$ & NA & NA & 3.2884 & NA & NA & NA \\
\hline 79 & 120 & 0.5 & 0.1 & $3,2,10$ & NA & NA & NA & NA & NA & NA \\
\hline 80 & 120 & 0.5 & 0.2 & $3,2,5$ & NA & NA & NA & NA & NA & NA \\
\hline 81 & 120 & 0.5 & 0.5 & $3,2,2$ & NA & NA & NA & NA & NA & NA \\
\hline 82 & 180 & 0.1 & 0.1 & $2,10,10$ & NA & NA & NA & NA & NA & NA \\
\hline 83 & 180 & 0.1 & 0.2 & $2,10,5$ & $\mathrm{NA}$ & NA & 4.033 & $\mathrm{NA}$ & NA & NA \\
\hline 84 & 180 & 0.1 & 0.5 & $2,10,2$ & 35.6142 & 10.2485 & 3.7688 & NA & NA & NA \\
\hline 85 & 180 & 0.2 & 0.1 & $2,5,10$ & NA & NA & NA & NA & NA & 1.7708 \\
\hline 86 & 180 & 0.2 & 0.2 & $2,5,5$ & NA & NA & NA & NA & NA & NA \\
\hline 87 & 180 & 0.2 & 0.5 & $2,5,2$ & NA & NA & 2.9226 & NA & NA & NA \\
\hline 88 & 180 & 0.5 & 0.1 & $2,2,10$ & NA & NA & NA & NA & NA & NA \\
\hline 89 & 180 & 0.5 & 0.2 & $2,2,5$ & NA & NA & NA & NA & NA & NA \\
\hline 90 & 180 & 0.5 & 0.5 & $2,2,2$ & NA & NA & NA & NA & NA & NA \\
\hline
\end{tabular}

\section{REFERENCES}

[1] .Abdel hamidAbdesselam, HuiHui Wang, and ArayananKulathuramaiyer - "Spiral Bit-string Representation of Colour for Image Retrieval"

[2] M.BabuRao, Dr. B.PrabhakaraRao\& Dr. A.Govardhan Apr 2011 - "Content Based Image Retrieval Using Dominant Colour, Texture And Shape" - International Journal of Engineering Science and Technology (IJEST), Vol. 3 No. 4 ISSN : 0975-5462

[3] Bing Wang -2008 - "A Semantic Description For Content-Based Image Retrieval" - at College Of Mathematics And Computer Science, Hebei University, Baoding 071002, China

[4] Bo Di - 2007 - "An efficient image retrieval approach base on Colour clustering" at Third International Conference on Intelligent Information Hiding and Multimedia Signal Processing,. IIHMSP

[5] Hafner, J and Sawhney, H. S.-1995 -. Efficient colorhistogram indexing for quadratic form distancefunctions.In IEEE Transactions on Pattern Analysis and Machine Intelligence, Intelligence, 17(7): pp.729-736.
[6] Ch.Kavitha, Dr. B.PrabhakaraRao\& Dr. A.Govardhan Feb 2011 - "An Efficient Content Based image Retrieval Using Colour And Texture Of Image Subblocks" in International Journal of Engineering Science and Technology (IJEST), Vol. 3 No. 2, ISSN : 0975-5462

[7] Ch.Kavitha, Dr. B.PrabhakaraRao\& Dr. A.Govardhan February 2011.- "Image Retrieval Based On Colour and Texture Features of the Image Sub-blocks" in International Journal of Computer Applications (0975 8887)Volume 15- No.7,

[8] ManimalaSinglia andK.Hemacllandran - 2011"Performance analysis of Colour Spaces In Image Retrieval" in Assam University Journal of Science \& Technology: Physical Sciences and TechnologyVol. 7 Number 11 94-104. : ISSN 0975-2773

[9] Muhammad Riaz, Kim Pankoo and Park Jongan - 2009 "Extracting Colour Using Adaptive Segmentation for Image Retrieval" in International Joint Conference on Computational Sciences and Optimization

[10] S.Niranjanan and S.P.RajaGopalan - January 2012 "Performance Efficiency of Quantization using HSV Colour Space And Euclidean Distance in CBIR" in International Journal of Action Research \& Engineering 
to Eradicate Poverty" Vol. No. 03, Issue No. 01. Pages $24-32$.

[11] S.Niranjanan and S.P.RajaGopalan - March 2012 "Performance Efficiency of Quantization using HSV Colour Space And Intersection Distance in CBIR" in International Journal of Computer Applications (0975 8887 ) Volume 42 - No. 21, Pages 48 - 55.

[12] RajshreeDubey, RajnishChoubey and SanjeevDubey June 2011 - "Efficient Image Mining using Multi Feature Content Based Image Retrieval System" in IntJr of Advanced Computer Engineering and Architecture Vol. 1, No. 1 ,

[13] .SangohJeong -Mar.15, $2001 \quad$ - "Histogram-Based Colour Image Retrieval"

[14] .Smith, J. R. - 1997 -. Integrated spatial and feature image system: Retrieval, analysis and compression, Ph.D dissertation, Columbia University, New York

[15] Vishal Chitkara - May 2001 - "Colour-Based Image Retrieval Using Compact Binary Signatures" in Technical Report TR 01-08 Department Of Computing Science, University of Alberta Edmonton, Alberta, Canada
[16] Wan, X and Kuo, - 1996 - Image retrieval with multiresolution color space quantization. inElectron.Imaging and Multimedia Syst.

[17] Wan. X and Kuo. K. - 1996 -. Color distribution analysisand quantization for image retrieval. in SPIE Storageand Retrieval for Image and Video Databases IV, vol.SPIE 2670, pp. 9- 16

[18] WaqasRasheed - 2008 - "Sum of Values of Local Histograms for Image retrieval" at Chosun University, Gwangju, South Korea

[19] Wasim Khan, Shiv Kumar, Neetesh Gupta, Nilofar Khan - March 2011 - "Signature Based Approach For Image Retrieval Using Colour Histogram And Wavelet Transform" in International Journal of Soft Computing and Engineering (IJSCE) Volume-1, Issue-1

[20] Zhang, Z., Wenhui, Land Bo, L. - 2009 -. An Improving Technique of Col or Histogram in Segmentation-based Image Retrieval. At FifihInternational Conference on Information Assurance and Security. IEEE. 